\title{
44 \\ CIN: An Intelligent Information Management Toolkit
}

\author{
G. Antoniou ${ }^{a}$, and M.A. Williams ${ }^{b}$
}

${ }^{a}$ School of Computing \& Information Technology

Griffith University, QLD 4111, Australia

ga@cit.gu.edu.au

${ }^{b}$ Information Systems, Dept. of Management

The University of Newcastle, NSW 2308, Australia

maryanne@frey.newcastle.edu.au

\begin{abstract}
Information systems are often faced with incomplete information, and as a consequence they have to make plausible conjectures to operate in a satisfactory way. A simple example is the Closed World Assumption which is used extensively in database systems. Default Reasoning provides formal methods which support such behaviour; the plausible conjectures are made in the absence of complete information based on default rules ("rules of thumb").

Information is subject to change due to the inherent uncertainty of information, or because the environment is volatile and dynamic. Current default reasoning systems neglect the problem raised by change. Belief Revision is the research area that has developed techniques capable of dealing with incomplete information.

This paper presents the motivations, the design decisions, and the current state of the CIN Project (Changing and Incomplete information). The aim of the project is to provide an integrated toolkit for intelligent information management. We believe that providing a toolkit which is open to future enhancements is important. It is our contention that there is no "best" method for default reasoning and belief revision. Instead the system developer must seek to determine the most appropriate method for the problem at hand.
\end{abstract}

Keywords

Intelligent Information Management, Incomplete Information, Changing Information 


\section{MOTIVATIONS AND AIMS OF CIN}

This paper presents the motivations, the design decisions and the current state of the CIN Project whose aim is to develop an integrated, domain-independent toolkit of default reasoning and belief revision methods.

The performance of an information system depends heavily upon the information it has at hand about the problem under consideration. Complete information is difficult to come by, and is generally not available even for information systems developed for simple database applications. For instance, database systems use the closed world assumption and introduce null values to deal with incomplete information. In general, an intelligent information system must be able to make plausible conjectures, which may turn out to be incorrect when more information becomes available. Nonmonotonic reasoning provides methods for making such conjectures when faced with incomplete information. In the Default Logic of Reiter (1980), for example, conjectures are made using rules of thumb (called default rules); see Figure 1.

Information is subject to change due to the inherent uncertainty of information or because the environment is volatile and dynamic. Belief Revision (Alchourron et al. (1985)) is the research area that has developed techniques capable of dealing with changing information (see Figure 2).

The CIN Project aims to make advanced techniques from Nonmonotonic Reasoning (NMR) and Belief Revision (BR) more accessible to information system developers. It will deliver an Intelligent Information Management Toolkit which provides the user with a suite of methods that can be used to solve real-world problems. The reason we design an open system is our contention that finding the right method for NMR and BR is an elusive dream, and that we should instead seek to determine the most appropriate method for the specific problem at hand. Some innovative aspects of the project include the following.

- Combination with software engineering methods: Intelligent information systems can benefit from lessons learnt in classical computer science. Software engineering techniques such as a modularity constructs on the knowledge level will enable the user to make a more effective use of the system. Our ambitious aim in this direction is to achieve a full combination of nonmonotonic reasoning and object-oriented techniques on the representational level.

- Integration: The current state of the art in nonmonotonic reasoning is that once a change in the knowlegde base, however minor, is performed, nonmonotonic reasoning must recalculate plausible conclusions from scratch; this is completely inappropriate, especially given that nonmonotonic reasoning is computationally hard. Our solution uses belief revision to overcome this problem.

The system is currently founded on the tableau-based theorem prover of Wallace (1994) written in $\mathrm{C}++$. It has been designed using object-oriented techniques, and makes aggressive use of parameterized typing, algorithm and container abstraction techniques, as well as iterators and adaptors. The theorem prover uses efficient abstractions that are of wider use in the development of the Toolkit. 


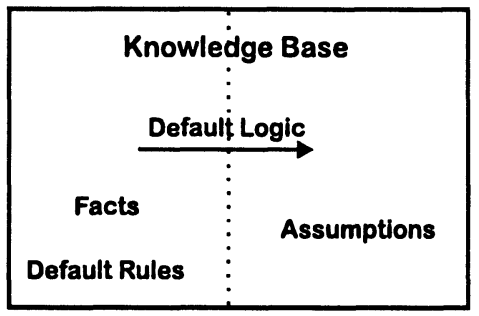

Figure 1 Default Logic allows defeasible assumptions to be deduced

\section{DEFAULT REASONING IN CIN}

In default reasoning there are two kinds of information:

- certain information given in the form of classical logical sentences (called facts). These may be observations that have been made or information known to be true ("Canberra is the capital city of Australia"), and

- default rules which describe plausible conclusions that can be made in the absence of information to the contrary. Information derived from the default rules is referred to as an assumption. Default rules are used to enrich the certain information by introducing information which is plausible but may turn out to be wrong.

Here are some examples for default rules: "Birds typically fly". Formally it is written as $\frac{\operatorname{bird}(X): f l i e s(X)}{\text { flies }(X)}$. This sort of default rule is called a typicality default because it describes a common case. There are other sorts of default rules, for example "Suspects are assumed innocent unless proven guilty." Formally written: $\frac{\text { suspect }(X): \text { innocent }(X)}{\text { innocent }(X)}$. Even if it is likely that a person is guilty, we prefer to decide in favour of the less likely case if it is plausible to assume that the person may be innocent (meaning that his guilt was not proven beyond reasonable doubt). Finally, an example from law: A general rule of German law is that foreign criminals are expelled.

$$
\frac{\operatorname{criminal}(X) \wedge \text { foreigner }(X): \text { expell }(X)}{\operatorname{expell}(X)} .
$$

Political refugees are a class of foreigners who cannot be expelled under any circumstancies. Such information is represented by a fact, e.g. foreigner $(X) \rightarrow \neg \operatorname{expell}(X)$.

The meaning (semantics) of a default theory is given in terms of so-called extensions which represent maximal alternative world views which are based on the given information. They are computed using the certain information and the default rules; extensions include all facts and a selection of assumptions. In general, default rules that conflict with one another lead to alternative extensions. So, if we know that a person is a Green and 


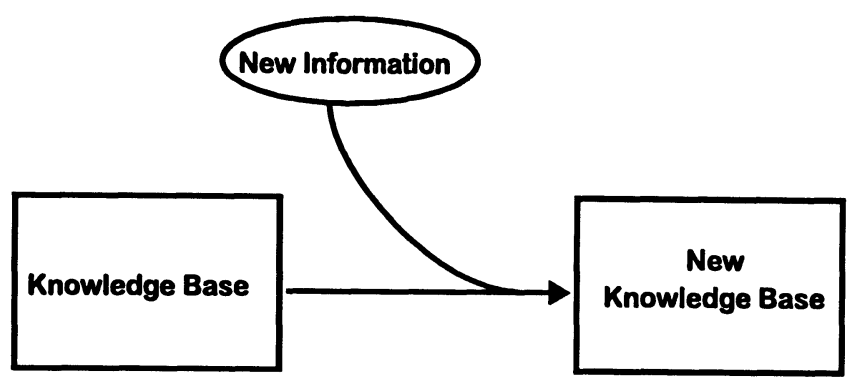

Figure 2 Belief Revision supports the incorporation of new information

an AAA member, then we might use either of the following default rules:

Greens typically don't like cars.

AAA members typically like cars.

Then we can derive conclusions that contradict each other by generating two separate extensions; one in which we would conclude that the person likes cars, and one in which we conclude that he does not. In fact, one of the advantages of default reasoning is that we can maintain conflicting pieces of information in the same information base without running into the problems of classical logic (anything can be concluded from an inconsistent knowledge base).

The basic functionality of the default reasoning component of the CIN system is to provide the user with the so-called extensions, which are world views that are compatible with and based on the given information; extensions include all facts and a selection of assumptions.

There exist several ways to define extensions. The original one was introduced in Reiter (1980), but many more have been developed since. For example the approaches of Lukaszewicz (1988), Delgrande et al. (1994), and Mikitiuk\&Truszczynski (1995). In general it is impossible to say that one variant is superior to all others; rather we have to choose the most appropriate one from application to application. In the spirit of a toolkit our system offers several of these possibilities to the information modeller, and leaves it up to them to determine which method is better suited to the particular problem at hand.

The efficiency of the default reasoning system is significantly enhanced by the use of search tree pruning techniques, intelligent use of a theorem prover, and stratification concepts of Cholewinski (1995). The system also offers explanation features at various levels of abstraction. Extensive technical information about the implementation of the default reasoning component can be found in Courtney et al. (1996). 
It should be noted that default reasoning was implemented in the early days of nonomonotonic reasoning in the form of truth maintenance systems, which can be also used to model the dynamics of information. Our system is superior to Truth Maintenance Systems (TMS) for two major reasons. Firstly TMS keeps a record of all interdependencies among the pieces of information. For realistic purposes this creates an enormous overhead; such bookkeeping is unnecessary in our approach. Furthermore, TMS's offer a low level of abstraction, whereas the solutions we offer to the problem of reasoning with changing and incomplete information have a clear declarative flavour.

\section{BELIEF REVISION IN CIN}

Belief revision provides operators that support the incorporation of new information into a knowledge base such that consistency is preserved. The basic functionality of the belief revision component of the CIN system is given the following:

- the current knowledge base $K$ with an associated preference ranking where the rank of a sentence represents its degree of belief, and

- some new information in the form of a sentence $s$ and its desired new rank $i$.

The belief revision system recalculates the ranks the contents of $K$ taking the new information into account. The recalculation is performed in accordance with the Principle of Minimal Change, i.e. the relative ranking of information in $K$ is modified as little as is necessary to accomodate the new information.

Potential application areas of Belief Revision include Marketing Research, HumanComputer Interaction, Diagnosis, and Software Engineering.

The belief revision system is an implements operators of the AGM paradigm which was developed by Alchourron, Gärdenfors and Makinson. It provides operators for modeling the revision and contraction of information. The principal constructions of these operators rely on some form of underlying preference ranking, such as the epistemic entrenchment ordering of Gärdenfors and Makinson (1988). An epistemic entrenchment ordering is a total preorder on the sentences in the language, e.g. the statement bird(Tweety) may have a higher epistemic entrenchment rank than flies(Tweety) depending on the system's background knowledge. According to the AGM paradigm this ordering can uniquely determine how the system will react to the intrusion of new information.

Adjustments were proposed by Williams (1994) as a way of realising the principle of an absolute measure of minimal change. The computational model for adjustments described in Williams (1995) forms the basis of the belief revision component of the CIN system. Lossely speaking an adjustment is a procedure that modifies a ranking by moving sentences up the ranking (revision) and sentences down the ranking (contraction).

The underlying idea of the implementation is to assign to each rank a layer object which encapsulates the tableau corresponding to the sentences included in that layer. Two of the entry points offered provide a means to locate the sentences entailed, and whether a particular sentence is entailed. The use of a tableau-based theorem prover allows the reuse of unsuccessful proofs; a significant feature that enhances the efficency of the system substantially. The architecture of the system is based on a collection of highly 
efficient parameterized types (vectors, lists, maps). Vectors are containers that arrange elements of a given type in a strictly linear arrangement and provide constant time access to any element. Lists are not random-access containers, but are optimized for frequent insertions and deletions in the middle of the sequence. A map is an associative container that supports unique keys of a given type, and provides for fast retrieval of values of another type based on the stored keys.

The algorithm is based on the calculation of degrees of acceptance for both explicit and implicit information. The system builds a tableau in a step-wise fashion reusing the previous layer's tableau to determine the degree of acceptance for implicit information. In this way the architecture of the system enhances the process of adjusting the ranking to incorporate new (possibly inconsistent) information.

\section{CURRENT AND FUTURE WORK}

The basic default reasoning and belief revision cells of CIN have been completed. The next steps in the development of the CIN system include the implementations for goaldriven query evaluation in default logic and its variants, and for several other strategies for belief revision such as the new reason-based procedure; maxi-adjustment (Williams, 1996). One of our current undertakings seeks to integrate default reasoning with belief revision operators. Future features of the CIN system include modular concepts, object-oriented information design, an extensive explanation component, and a hypothetical reasoning capability for "what if" analysis.

We are also investigating and preparing several applications. In particular, we have identified the following domains as interesting for our purposes:

- Marketing Research, in particular modeling changes in consumer preferences. Modeling consumer preferences of a product class involves the collection of data and culminates in a ranking that reflects the consumers preferences. Conjoint analysis is then used to compute utility functions that represent the consumers willingness to trade off varying levels of product attributes on the basis of their preferences. Williams and Polonsky (1996) proposed an integrated framework for conjoint analysis and belief revision as a means to circumvent the need for data collection (a labour intensive and expensive activity) when consumers change their preferences.

- Requirements Analysis, in particular the study of the effects of changes in user requirements in software engineering. MacNish and Williams (1996) provide a formal framework for modeling design revision using belief revision techniques.

- Legal reasoning. Laws are full of rules with exceptions, therefore default rules are an appropriate representation of legal rules.

- Intelligent Decision Support Systems for negotiations in many business areas, in pretrial agreements, in industrial relations etc.

\section{REFERENCES}

C. Alchourron, P. Gardenfors and D. Makinson (1985) On the Logic of Theory Change: 
Partial Meet Functions for Contraction and Revision. Journal of Symbolic Logic, 50, 510-530.

G. Antoniou (1996) Nonmonotonic reasoning with incomplete and changing information. MIT Press.

P. Cholewinski (1995) Reasoning with stratified default theories. In Proc. LPNMR'95, Springer LNAI 928, 456-470.

A. Courtney, G. Antoniou and N. Foo (1996). Exten: A System for Computing Default Logic Extensions. In Proc. 4th Pacific Rim International Conference on Artificial Intelligence (PRICAI'96), Springer LNAI (in press)

J.P. Delgrande, T. Schaub and W.K. Jackson (1994) Alternative approaches to default logic. Artificial Intelligence, 70, 167-237.

P. Gärdenfors and D. Makinson (1988) Revisions of Knowledge Systems using Epistemic Entrenchment. In the Proceedings of the 2nd Conference on Theoretical Aspects of Reasoning About Knowledge, 83-96.

W. Lukaszewicz (1988) Considerations of default logic: an alternative approach. Computational Intelligence, 4,1, 1-16.

A. Mikitiuk and M. Truszczynski (1995) Constrained and rational default logics. In Proc. 14th International Joint Conference on Artificial Intelligence, 1509-1515.

C.K. MacNish and M.A. Williams (1996), From Belief revision to design revision: Applying theory change to changing requirements, Pacific Rim Workshop on Incomplete and Changing Information, (in press).

R. Reiter (1980) A logic for default reasoning. Artificial Intelligence, 13, 81-132.

K. Wallace (1994), Proof truncation techniques in model elimination tableau-based theorem provers, PhD Dissertation, University of Newcastle.

M.A. Williams (1994), Transmutations of Knowledge Systems, in J. Doyle, E. Sandewall, and P. Torasso (eds), Proceedings of the Fourth International Conference on Principles of Knowledge Representation and Reasoning, Morgan Kaufmann, 619 - 629.

M.A. Williams (1995), Iterated Theory Base Change: A Computational Model. In the Proceedings of the Fourteenth International Joint Conference on Artificial Intelligence, Montréal, 1541-1550.

M.A. Williams (1996), A commonsensical approach to belief revision, in the Proceedings of the Symposium on Common Sense, Stanford University, 245 - 262.

M.A. Williams and M.J. Polonsky (1996), Modeling changing consumer preferences using belief revision, in the Proceedings of the Fourth International Workshop on Artificial Intelligence, Economics and Management, (in press).

M.A. Williams, K. Wallace, G. Antoniou (1995) An Object-Oriented Implementation of Belief Revision. In the Proceedings of the 8th Australian Joint Conference on Artificial Intelligence, 259-266. 